\title{
BMJ Open Assessing the hospital volume-outcome relationship in surgery: a scoping review protocol
}

\author{
Mathieu Levaillant (D) , ${ }^{1}$ Romaric Marcilly, ${ }^{2}$ Lucie Levaillant, ${ }^{3}$ Benoît Vallet, ${ }^{1}$ \\ Antoine Lamer ${ }^{1}$
}

To cite: Levaillant $\mathrm{M}$, Marcilly $\mathrm{R}$, Levaillant $\mathrm{L}$, et al. Assessing the hospital volume-outcome relationship in surgery: a scoping review protocol. BMJ Open 2020;10:e038201. doi:10.1136/ bmjopen-2020-038201

- Prepublication history for this paper is available online. To view these files, please visit the journal online (http://dx.doi. org/10.1136/bmjopen-2020038201).

Received 03 March 2020 Revised 02 September 2020 Accepted 13 September 2020

A Check for updates

(C) Author(s) (or their employer(s)) 2020. Re-use permitted under CC BY-NC. No commercial re-use. See rights and permissions. Published by BMJ.

${ }^{1}$ Univ. Lille, CHU Lille, ULR 2694 - METRICS : Évaluation des technologies de santé et des pratiques médicales, F-59000 Lille, France

${ }^{2}$ Univ. Lille, CHU Lille, ULR 2694 - METRICS : Évaluation des technologies de santé et des pratiques médicales, INSERMCIC-IT 1403/Evalab, F-59000 Lille, France

${ }^{3}$ Department of Pediatric Endocrinology and Diabetology, University Hospital Centre Angers, Angers, Pays de la Loire, France

Correspondence to

Mathieu Levaillant;

mathieu.levaillant@gmail.com

\section{ABSTRACT}

Introduction Even if a positive volume-outcome correlation in surgery is mostly admitted in many surgical fields, the various ways to assess this relationship make it difficult for researchers and policymakers to use it. Our aim is therefore to provide an overview of the way hospital volume-outcome relationship was assessed. Through this overview, our goal is to identify potential gaps in the assessment of this relationship, to help researchers who want to pursue work in this field and, ultimately, to help policy makers interpret such analyses.

Methods and analysis This review will be conducted using the six stages of the scoping review method: identifying the research question, searching for relevant studies, selecting studies, data extraction, collating, summarising and reporting the results and concluding. This review will address all the key questions used to assess the volume-outcome relationship in surgery. Primary research papers investigating the hospital volumeoutcome relationship from 2009 will be included. Studies only looking at surgeons' volume-outcome relationship or studies were the volume variable is not individualisable will be excluded.

Both MEDLINE and Scopus will be searched along with grey literature. Two researchers will perform all the stages of the review: screen the titles and abstracts, review the full text of selected articles to determine final inclusions and extract the data. The results will be summarised quantitatively using numerical counts.

Ethical considerations and dissemination Reviews of published articles are considered secondary analysis and do not need ethical approval. The findings will be disseminated through multiple channels like conferences and peer-reviewed journals.

\section{INTRODUCTION}

The hospital volume-outcome relationship has been discussed for years, especially in surgical disciplines. Several primary studies indicate a positive relationship between hospital volume and clinical outcomes for different surgical procedures: ${ }^{1-4}$ indeed, the higher the operative volume, the better the patient's outcomes (mainly the mortality). The positive volume-outcome relationship led researchers to recommend the creation of thresholds of surgeries in order to limit

\section{Strengths and limitations of this study}

This scoping review will identify gaps in the assessment of the hospital volume-outcome relationship and provide future researchers with an overview of how this relationship was assessed.

- This review aims ultimately to help policy makers interpret pragmatically the volume-outcome relationship analyses based on the methodology chosen in order to help make health policy decision.

- The protocol is rigorous, the search strategy broad, and includes both peer-reviewed and grey literature, and data extraction process is clearly described.

- Quality of the papers studied was not assessed as this is a protocol for a scoping review and aims to provide an overview of the volume-outcome assessment methodology.

- By limiting our search to original English articles, we will exclude some potentially important results in other kinds of studies or languages.

centres with low activity ${ }^{567}$ These conclusions are consistent with the ones already drawn by scientific authorities as the Expert panel on Weight Loss Surgery for bariatric surgery, ${ }^{8}$ or with policies implemented in a few countries such as France in 2007. These thresholds must be taken into account by policymakers when they organise the sanitary map.

Even if volume-outcome relationship is confirmed, Morch et al's recent systematic review pointed out the shortcomings in methodologies and suggested further research, with special attention to the methodology specific to volume-outcome relationship. ${ }^{4}$ Virtually, all papers exploring the relationship used their own method: sometimes surgeon's volume is investigated and sometimes hospitals', often as a categorical value but occasionally as a continuous one. The differences in the methodologies used can have direct consequences on the conclusion of a positive volume-outcome relationship. ${ }^{9} 10$ This disparity of methods makes it difficult to compare these research papers with each 
other and might complicate policymakers' decisions. Even more, the way the relationship is assessed seems sometimes irrelevant. For example, considering volume as a quantitative variable implies that all the centres, except the biggest one, increase the mortality risk. The covariates used to assess the relationship can also have a major impact on the interpretation of the results. A recent study about volume-outcome relationship following cholangiocarcinoma resection showed that when adjusting the volume-effect on the travel distance, this effect was not anymore significant, ${ }^{11}$ showing the importance of choosing the relevant covariates. All the aspects of the assessment of a volume-outcome relationship must be taken into account by policymakers ${ }^{12}$ if they want to structure the necessary geographic mapping.

Moreover, even if high volume is associated with better outcomes across a wide range of procedures and conditions, the magnitude of the association varies greatly. Indeed, even if the volume-outcome correlation is identified, the way to set thresholds and to study this association is facing a lot of interrogation and confronting new issues. This impacts the capacity for policymakers to take this relationship as reliable information and modify the health organisation based on it.

In order to have useful and relevant information, studies should not look only for the link between outcome and hospital volume, but they should also stratify it on patients' overall health condition. Because high-volume centres are often large hospitals with specialised teams, assessing this particular link should enable sorting patients on their original seriousness in order to identify the specific impact of volume on patient outcome. It will prevent the bias of evaluating the patient recruitment more than the volume-outcome. ${ }^{13}$

Finally, although mortality is an essential outcome, it cannot be the only one sought for assessing whether a centre should be closed. According to the type of surgery explored, studies have shown that even with no effect on mortality, volume can have an impact on the length of stay of the patient, ${ }^{12}$ the time to recovery, ${ }^{14}$ cost of the stay, ${ }^{15}$ related morbidity ${ }^{16}$ or even disease-free survival ${ }^{18}$

${ }^{19}$ for oncological surgeries.

All of these elements are in favour of looking at the volume-outcome relationship in a wider way, including more than just the mortality outcome or exploring volume as a continuous variable.

Our aim is therefore to provide an overview of how hospital surgical volume-outcome relationship was assessed through the key information used: outcomes explored, covariates included in the model, statistical analysis performed, gravity's assessment and so on. Through this overview, our goal is to identify potential gaps in this relationship's assessment to help researchers who want to pursue work in this field and, ultimately, to help policymakers in the interpretation of such analyses. With this goal in mind, we decided to investigate how the hospital volume-outcome relationship was assessed in studies through a scoping review, which is a form of knowledge synthesis that addresses an exploratory research question aimed at mapping key concepts, types of evidence and gaps in research. ${ }^{20} 21$

\section{METHODS AND ANALYSIS}

This protocol provides the essential procedures for conducting the review, including search strategy and articles' selection, as well as steps in analysing the obtained articles.

This scoping review method will be conducted using the six stages developed by Arksey and O'Malley ${ }^{22}$ with recent advancements by Levac et $a l^{23}$ and will be reported in accordance with Preferred Reporting Items for Systematic reviews and Meta-Analyses extension for Scoping Reviews (PRISMA-ScR) guidelines. ${ }^{24}$

\section{Step 1: identifying the research question}

To meet the objectives of the study, researchers substantiated the research question:

How hospital surgical volume-outcome relationship is assessed?

This question was developed through the following ones:

- What databases were used, and what frame of reference was used to explore it?

- Which surgery and surgical disciplines were explored? What were the inclusion and exclusion criteria?

- How was the statistical analysis performed? How was the hospital-volume variable used? What kind of

Table 1 Inclusion and exclusion criteria for surgical volume-outcome relationship relevant studies

\begin{tabular}{|c|c|c|c|}
\hline Population & Concept & Context & Types of sources \\
\hline $\begin{array}{l}\text { Inclusion: studies about } \\
\text { surgical hospital volume- } \\
\text { outcome relationship with } \\
\text { a sufficiently described } \\
\text { methodology to be able to } \\
\text { extract all data sought } \\
\text { Exclusion criteria: only } \\
\text { surgeon-specific volume- } \\
\text { outcome relationship }\end{array}$ & $\begin{array}{l}\text { Inclusion: every methodology } \\
\text { used to assess surgical } \\
\text { volume outcome } \\
\text { Exclusion: study including } \\
\text { the hospital volume only as a } \\
\text { covariate in a model in which } \\
\text { the impact of volume itself } \\
\text { was not clearly identifiable }\end{array}$ & $\begin{array}{l}\text { Inclusion: any type of surgery } \\
\text { Any type of patient-related } \\
\text { outcome (length of stay, } \\
\text { mortality, morbidity, cost, ...) } \\
\text { Any countries } \\
\text { No exclusion }\end{array}$ & $\begin{array}{l}\text { Inclusion: only primary } \\
\text { quantitative studies will be } \\
\text { included, written in English } \\
\text { and published between } 2009 \\
\text { and the data extraction date } \\
\text { Exclusion: other designed } \\
\text { studies }\end{array}$ \\
\hline
\end{tabular}


Table 2 Keywords and query used for MEDLINE and Scopus

\begin{tabular}{|c|c|}
\hline Database & Keywords and query \\
\hline MEDLINE & $\begin{array}{l}\text { Keywords: Volume, outcome, hospital, surgery, surgical, mortality, morbidity and cost } \\
\text { Query: ("Volume-outcome" OR "Volume-mortality" OR ("hospital volume" AND ("outcome" OR "mortality" OR } \\
\text { "morbidity" OR "cost"))) AND ("surgery" OR "surgical") AND "hospital" NOT "surgeon" (TITLE) }\end{array}$ \\
\hline Scopus & 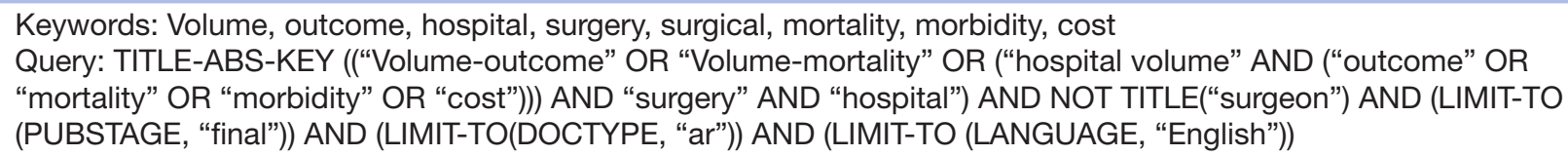 \\
\hline
\end{tabular}

covariates were included in the model and how was the initial patient's severity assessed? Which tests were used?

- How were the results presented and did the study conclude in a positive volume-outcome relationship?

\section{Step 2: identifying relevant studies}

The review follows the Joanna Briggs Institute (JBI) methodology $^{25}$ to identify the suitability of articles (table 1 ).

According to JBI's recommendations, both published and unpublished primary studies should be screened using the following three steps.

First, we will conduct a search on MEDLINE and Scopus databases for articles about our research topic, using "volume-outcome" or "hospital volume" keywords and look through them for the keywords used in December 2019.

After screening these articles, we will use the following keywords for the definitive query: "volume", "hospital", "mortality", "morbidity", "cost", "outcome", "surgery" and "surgical" (table 2), followed by analysis of the words contained in titles, abstracts, keywords and index terms to find related keywords and index terms.

After including articles, we will look for additional studies from the reference lists of all included studies and from other sources founded through personal researches. We will also search for grey literature to identify unpublished materials by using the Google Scholar database.

\section{Step 3: study selection}

The review will be conducted by two reviewers at both stages, one is a resident in public health (ML) and the second is a doctor in medical informatics (AL). They both participated in writing the study protocol. The two reviewers will perform all the screening stages independently.

First, the literature will be screened by title and abstract according to the inclusion and exclusion criteria (table 3). Studies will be included if they have both inclusion criteria and none of the three exclusion ones.

All the papers included by either one of the reviewers will go through the next step to ensure not to exclude an interesting paper.

Finally, the full text of the articles which passed the first stage will be assessed for inclusion (figure 1). If the reviewers disagree on an article, a third reviewer who participates on elaborating the study protocol will be involved to definitively constitute the study sample.

\section{Step 4: data extraction}

The data extraction will include the following key information about each study: author, year of publication, research design, research objectives, published or grey literature.

Details of the methodology used to assess the volumeoutcome relationship will be extracted based on the principles below and according to the extraction form (table 4). The database used will be sorted into six categories as recommended by the University of Washington: electronic health record, administrative data, claims data, patient or disease register, health surveys or clinical trials data. ${ }^{26}$ Studied surgeries will be addressed according to the 13 subspecialities proposed to French doctors during their initial training. ${ }^{27}$ The use of International Classification of Disease to identify the study population will be screened. Inclusion and exclusion criteria are going to be noted down, and uncommon criteria will be reported in the final review. Only a narrative description will be provided for this part.

Statistical analysis method will be pointed out, such as the statistical method described by Yan $e t a l^{28}$ and the way of presenting the results will be classified between graphs, tables or both. Graphs will be addressed as scatter gram, line graph, bar graph, histogram, pie chart, box-plot or

\begin{tabular}{|c|c|}
\hline Criteria & Review result \\
\hline \multicolumn{2}{|l|}{ Inclusion } \\
\hline $\begin{array}{l}\text { Surgical hospital volume-outcome } \\
\text { relationship }\end{array}$ & $\square$ Yes $\square$ No \\
\hline $\begin{array}{l}\text { Methodology used precisely described (how } \\
\text { the outcome was assessed, how the hospital } \\
\text { volume was analysed and how the statistical } \\
\text { analysis was performed). }\end{array}$ & $\square$ Yes $\square$ No \\
\hline \multicolumn{2}{|l|}{ Exclusion } \\
\hline Surgeon volume-outcome relationship only & $\square$ Yes $\square$ No \\
\hline Hospital volume used only as a covariate & $\square$ Yes $\square$ No \\
\hline $\begin{array}{l}\text { Publication in the form of systematic reviews, } \\
\text { qualitative studies, editorials, letters to the } \\
\text { editor, comments or narrative reports }\end{array}$ & $\square$ Yes $\square$ No \\
\hline
\end{tabular}




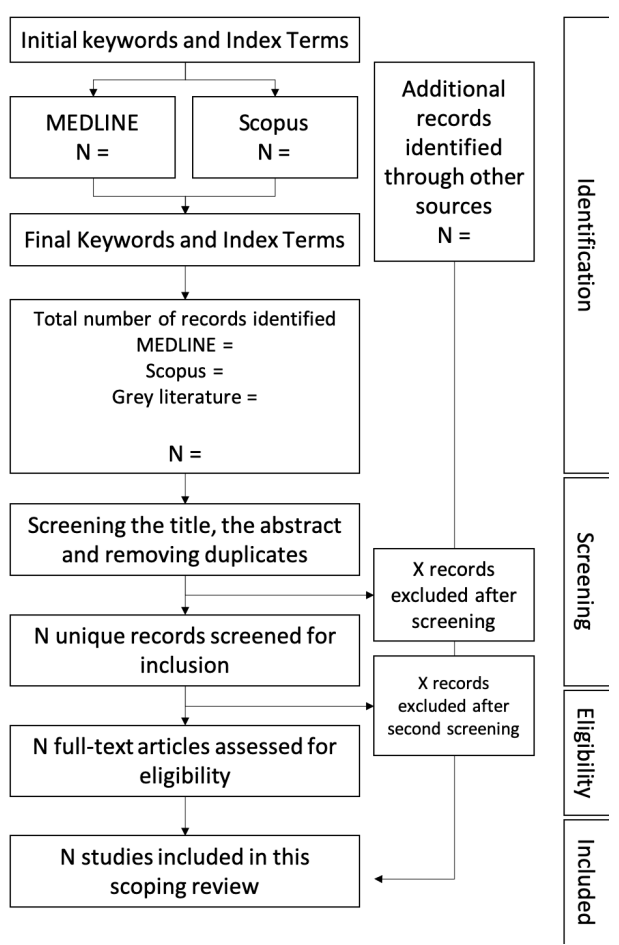

Figure 1 Flow chart for search strategy and study selection.

other, as described by Slutsky $e t a l .^{29}$ The way the hospital volume variable is assessed will be summarised between continuous, qualitative or both. For qualitative assessment, studies will be separated according to their type of hospital volume splitting: fractals, statistical cut-off points, arbitrary or literature thresholds, and other.

Outcomes will be extracted and classified according to the frequently observed categories in literature which are mortality, cost, length of stay, readmission or others. Others will be noted down and the most frequently observed will be quoted in the review paper, as a narrative analysis. Information about how the different studies stratified their analysis on initial patients' severity will be separated between the ones using known scores like Charlson Comorbidity Index adapted by Deyo, ${ }^{30}{ }^{31}$ Elixhauser score ${ }^{32}$ or other information. Covariates used to adjust the statistical models will also be listed and go through a narrative analysis in order to be exhaustive on what was used.

Finally, the study conclusion will be written down and sorted between positive and negative volume-outcome relationship.

Each reviewer will test the extraction data form (table 4) on the five first studies to ensure that all relevant results are extracted. If needed, the grid will be adjusted after the testing by both reviewers. Once the final grid is accepted by both the reviewers, all data will be extracted from all the papers by each of them.

\section{Stage 5: collating, summarising and reporting the results}

After collecting all the data, descriptive results will be summarised in one table, reporting each included article. A first part of the review will describe the articles included according to the country where the research was pursued, the journal that published the article and the year of publication. Surgeries explored and discipline concerned will also be described quantitatively. The number of databases used will be provided, along with their distribution among the six pre-identified categories.

Inclusion and exclusion criteria used in the included studies will be narratively described. The different criteria used will be summarised in a table, but no quantitative assessment will be provided.

Outcomes used will be listed along with the way they were quantitatively assessed. An analysis of the proportion of studies looking at each outcome over time will be conducted in order to identify how volume-outcome relationship research has evolved in recent years.

If possible, covariates will be listed and categorised (patient characteristics, hospital characteristics, gravity assessment, surgical precisions and so on). They will not be quantitatively assessed because our goal is to give an exhaustive overview of the variable used.

Statistical assessment, focussing on the volume-outcome relationship evaluation, will be analysed quantitatively. The most frequently observed methods will be described and precisions about them will be provided in order to help researchers to choose the most adapted method for their research question and help policymakers understand results provided in already published studies.

\section{Limitations}

This review may suffer from limitations.

The predefined categories may be insufficient to analyse every types of studies we will include. Indeed, categories of database of subspecialities of surgery chosen or statistical methods may be in unsuitable. To overcome this limitation, the extraction grid will be tested on five studies by both the reviewers and will be adapted if needed. Besides, the 'other' category will allow the management of unforeseen cases by allowing free text entry of these new cases.

The study is limited by the number of electronic databases that will be explored: only two electronic databases will be explored (MEDLINE and Scopus) along with grey literature. By caution, we will search through Google Scholar and look for additional studies from the reference lists of all the included studies. Even though, there is a possibility that relevant literature from other databases may be missed during our researches. ${ }^{33}$

Finally, to explore the entire surgical teams' effect on patients' outcome, we have chosen to explore only the hospital volume-outcome relationship through the scoping review. This choice implies that the results will be relevant only when exploring volume-outcome relationship from a general hospital perspective, and may not be similar to surgeon's volume-outcome relationship.

This review aims to propose a broad and accurate picture of how the volume-outcome relationship in surgery was assessed, by answering all the previously stated research questions. Also, it may identify gaps in the literature for future studies on this issue. Finally, considering the 
Table 4 Data extraction form

Title

Author

Year of publication

Research design

Research objectives

Type of sources

$\neg$ Published $\square$ Grey literature

Specific information

1. Database used

$\square$ Electronic health record $\square$ Administrative data

$\square$ Claims data $\square$ Patient or disease register

$\square$ Health survey $\square$ Clinical trials data

\section{Surgery studied}

$\square$ Head and Neck $\square$ Oral $\square$ Orthopaedics $\square$ Paediatrics $\square$ Plastics $\square$ Vascular

$\square$ Thoracic and cardiovascular $\square$ Visceral and digestive $\square$ Neurosurgery

$\square$ ENT $\square$ Obstetrics and gynaecology $\square$ Ophthalmology $\square$ Urology

\section{Use of the International Classification of $\square$ Yes $\square$ No}

Disease

4. Inclusion and exclusion criteria

5. Method to sort hospitals by volume

$\square$ Continuous $\square$ Categorical $\square$ Both

If continuous or both, precise the method used:

6. Outcomes
If other, precise the interesting outcome
studied
7. Severity score
and covariates

$\square$ Mortality $\square$ Cost $\square$ Length of stay

$\square$ Readmission $\square$ Other

8. Statistical methods

$\square$ Charlson/Deyo $\square$ Elixhauser $\square$ Other

$\neg$ One sample test $\square$ Two independent sample test

$\square$ Two correlated sample test $\square$ More than two independent sample test $\square$ More than two correlated sample test $\square$ Correlation $\square$ Two categorical variables $\square$

Same categorical outcome on matched pairs

$\square$ Linear multiple regression $\square$ Other

If other, precise

\section{9 .Results' presentation}

$\square$ Graph(s) $\square$ Table(s) $\square$ Both

If graph or both, its precise typology

$\square$ Line graph $\square$ Bar graph $\square$ Scatter gram $\square$ Histogram $\square$ Pie chart $\square$ Boxplot $\square$ Other

10. Conclusion: positive volume-outcome $\quad \square$ Yes $\square$ No relationship

meaning of the findings as they relate to the overall study purpose, and after discussion of advantages and disadvantages of each point, implications for future research practice and policy will be discussed.

\section{Patient and public involvement}

No patient involved.

\section{Ethical considerations and dissemination}

Reviews of published articles are considered secondary analysis and do not need ethical approval. This protocol reports a rigorous methodology.
This review aims to improve the way of assessing the volume-outcome relationship in surgery by summarising how it was already performed and by identifying gaps in knowledge and research. The findings will be disseminated through multiple channels like conferences and peer-reviewed journals and may help future research. The results may also add substantial data supporting new policies.

Twitter Mathieu Levaillant @MatLevaillant and Lucie Levaillant @LucieLevaillant 
Contributors ML led the design and conceptualisation of this work, and developed the search strategy with AL. ML and AL wrote the manuscript. RM served as an expert in designing the study protocol. RM, LL and BV provided feedback, finalised the manuscript and edited the final manuscript. All authors approved the final manuscript.

Funding The authors have not declared a specific grant for this research from any funding agency in the public, commercial or not-for-profit sectors.

Competing interests None declared.

Patient and public involvement Patients and/or the public were not involved in the design, or conduct, or reporting, or dissemination plans of this research.

Patient consent for publication Not required.

Provenance and peer review Not commissioned; externally peer-reviewed.

Open access This is an open access article distributed in accordance with the Creative Commons Attribution Non Commercial (CC BY-NC 4.0) license, which permits others to distribute, remix, adapt, build upon this work non-commercially, and license their derivative works on different terms, provided the original work is properly cited, appropriate credit is given, any changes made indicated, and the use is non-commercial. See: http://creativecommons.org/licenses/by-nc/4.0/.

\section{ORCID iD}

Mathieu Levaillant http://orcid.org/0000-0003-2124-9801

\section{REFERENCES}

1 El Amrani M, Clement G, Lenne X, et al. The impact of hospital volume and Charlson score on postoperative mortality of proctectomy for rectal cancer: a nationwide study of 45,569 patients. Ann Surg 2018;268:854-60.

2 El Amrani M, Clement G, Lenne X, et al. Failure-To-Rescue in patients undergoing pancreatectomy: is hospital volume a standard for quality improvement programs? nationwide analysis of 12,333 patients. Ann Surg 2018;268:799-807.

3 Pasquer A, Renaud F, Hec F, et al. Is centralization needed for esophageal and gastric cancer patients with low operative risk?: a nationwide study. Ann Surg 2016;264:823-30.

4 Morche J, Mathes T, Pieper D. Relationship between surgeon volume and outcomes: a systematic review of systematic reviews. Syst Rev 2016;5:204.

5 Dixon M, Mahar A, Paszat L, et al. What provider volumes and characteristics are appropriate for gastric cancer resection? Results of an international RAND/UCLA expert panel. Surgery 2013:154:1100-9.

6 Choi H, Yang S-Y, Cho H-S, et al. Mortality differences by surgical volume among patients with stomach cancer: a threshold for a favorable volume-outcome relationship. World J Surg Oncol 2017:15:134.

7 Gutacker N, Bloor K, Cookson R, et al. Hospital surgical volumes and mortality after coronary artery bypass grafting: using international comparisons to determine a safe threshold. Health Serv Res 2017;52:863-78.

8 Lim RB, Blackburn GL, Jones DB. Benchmarking best practices in weight loss surgery. Curr Prob/ Surg 2010;47:79-174.

$9 \mathrm{Kim} \mathrm{W}$, Wolff S, Ho V. Measuring the volume-outcome relation for complex Hospital surgery. Appl Health Econ Health Policy 2016;14:453-64.

$10 \mathrm{Yu}$ T-H, Tung Y-C, Chung K-P. Does categorization method matter in exploring volume-outcome relation? a multiple categorization methods comparison in coronary artery bypass graft surgery surgical site infection. Surg Infect 2015;16:466-72.

11 Beal EW, Mehta R, Hyer JM, et al. Association between travel distance, hospital volume, and outcomes following resection of cholangiocarcinoma. J Gastrointest Surg 2019;23:944-52.
12 Modrall JG, Minter RM, Minhajuddin A, et al. The surgeon volume-outcome relationship: not yet ready for policy. Ann Surg 2018;267:863-7.

13 Lee KCL, Sethuraman K, Yong J. On the hospital volume and outcome relationship: does specialization matter more than volume? Health Serv Res 2015;50:2019-36.

14 Balentine CJ, Naik AD, Robinson CN, et al. Association of highvolume hospitals with greater likelihood of discharge to home following colorectal surgery. JAMA Surg 2014;149:244-51.

15 Sutton JM, Hoehn RS, Ertel AE, et al. Cost-Effectiveness in hepatic lobectomy: the effect of case volume on mortality, readmission, and cost of care. J Gastrointest Surg 2016;20:253-61.

16 Gourin CG, Stewart CM, Frick KD, et al. Association of hospital volume with laryngectomy outcomes in patients with larynx cancer. JAMA Otolaryngol Head Neck Surg 2019;145:62-70.

17 Odagiri $\mathrm{H}$, Yasunaga $\mathrm{H}$, Matsui $\mathrm{H}$, et al. Hospital volume and adverse events following esophageal endoscopic submucosal dissection in Japan. Endoscopy 2017;49:321-6.

18 Borowski DW, Bradburn DM, Mills SJ, et al. Volume-Outcome analysis of colorectal cancer-related outcomes. Br J Surg 2010;97:1416-30.

19 van der Werf LR, Cords C, Arntz I, et al. Population-Based study on risk factors for Tumor-Positive resection margins in patients with gastric cancer. Ann Surg Oncol 2019;26:2222-33.

20 Grant MJ, Booth A. A typology of reviews: an analysis of 14 review types and associated methodologies. Health Info Libr J 2009;26:91-108.

21 Munn Z, Peters MDJ, Stern C, et al. Systematic review or scoping review? guidance for authors when choosing between a systematic or scoping review approach. BMC Med Res Methodol 2018;18:143.

22 Arksey H, O'Malley L. Scoping studies: towards a methodological framework. Int J Soc Res Methodol 2005;8:19-32.

23 Levac D, Colquhoun H, O'Brien KK. Scoping studies: advancing the methodology. Implement Sci 2010;5:69.

24 Tricco AC, Lillie E, Zarin W, et al. PRISMA extension for scoping reviews (PRISMA-ScR): checklist and explanation. Ann Intern Med 2018;169:467.

25 JBI. Chapter 11: Scoping reviews - JBI Reviewer's Manual - JBI Global Wiki. Available: https://wiki.joannabriggs.org/display/ MANUAL/Chapter+11\%3A+Scoping+reviews [Accessed 31 Jul 2019].

26 Maloy C. Library guides: data resources in the health sciences: clinical data. Available: https://guides.lib.uw.edu/hsl/data/findclin [Accessed 18 Feb 2018].

27 République française. Arrêté du 27 novembre 2017 modifiant l'arrêté du 12 avril 2017 relatif l'organisation du troisième cycle des études de médecine et l'arrêté du 21 avril 2017 relatif aux connaissances, aux compétences et aux maquettes de formation des diplômes d'études spécialisées et fixant la liste de ces diplômes et des options et formations spécialisées transversales du troisième cycle des études de médecine, 2017.

28 Yan F, Robert M, Li Y. Statistical methods and common problems in medical or biomedical science research. Int J Physiol Pathophysiol Pharmacol 2017;9:157-63.

29 Slutsky D. The effective use of graphs. J Wrist Surg 2014;03:067-8.

30 Charlson ME, Pompei P, Ales KL, et al. A new method of classifying prognostic comorbidity in longitudinal studies: development and validation. J Chronic Dis 1987;40:373-83.

31 Deyo RA, Cherkin DC, Ciol MA. Adapting a clinical comorbidity index for use with ICD-9-CM administrative databases. J Clin Epidemiol 1992;45:613-9

32 Elixhauser A, Steiner C, Harris DR, et al. Comorbidity measures for use with administrative data. Med Care 1998;36:8-27.

33 Bramer WM, Rethlefsen ML, Kleijnen J, et al. Optimal database combinations for literature searches in systematic reviews: a prospective exploratory study. Syst Rev 2017;6:245. 